\title{
Comparación entre el stent medicado Zilver PTX y la derivación con injerto sintético en el sector femoropoplíteo
}

\section{Comparison between drug-eluting stent Zilver PTX and infrainguinal revascularization with synthetic graft}

\author{
Rodrigo Garza-Herrera, Carlos R. Ramos-López, César Nuño-Escobar*, Francisco J. Llamas-Macías, \\ José T. R. Pulido-Abreu, Alejandro Celis-Jiménez y P. César Olvera-Hernández \\ Unidad Médica de Alta Especialidad, Centro Médico Nacional de Occidente, Instituto Mexicano del Seguro Social, Guadalajara, Jalisco, México
}

\begin{abstract}
Resumen
Objetivo: La terapia endovascular ofrece menor costo y reducción en la morbimortalidad. El uso del stent medicado Zilver PTX muestra resultados prometedores con permeabilidad del $89.9 \%$ a un año. El objetivo es analizar la permeabilidad a 6 meses y la presencia de complicaciones con el uso del stent medicado Zilver PTX en comparación a la derivación femoropoplítea sintética. Métodos: Estudio retrospectivo que abarca dos grupos de 15 pacientes cada uno, con una edad promedio de 66 años, todos presentan diagnóstico de enfermedad arterial periférica (EAP) sintomática. El grupo control fue sometido a revascularización con injerto sintético, mientras el grupo de estudio fue tratado con stent medicado Zilver PTX. Ambos grupos fueron vigilados por un periodo de 6 meses. Resultados: En comparación, ambos métodos son eficaces en el tratamiento de la EAP del sector femoropoplíteo. Para el grupo tratado con stent medicado, se encontró disminución en días de estancia intrahospitalaria y tendencia a incrementar la clase funcional. La permeabilidad primaria fue del 87 vs. el 93\% en la derivación abierta y grupo manejado con stent medicado Zilver PTX, respectivamente. Como permeabilidad secundaria se encontró $93 \%$ vs. 100\%. Conclusiones: Ambos métodos son eficaces para tratar la enfermedad femoropoplítea. Nuestros resultados favorecen el uso de stent medicado en comparación a derivación con injerto sintético.
\end{abstract}

Palabras clave: Stent liberador de droga. Zilver PTX. Injerto sintético. Revascularización sintética.

\section{Abstract}

Objective: Endovascular therapy offers reduction in costs, morbidity and mortality. Drug eluting stent (DES) Zilver PTX has up to $89.9 \%$ patency rates. Our objective is to determinate patency and presence of complications at 6 months with the DES Zilver PTX in comparison to open bypass using ePTFE in the infrainguinal segment. Methods: This is a retrospective study with two groups of 15 patients each, with a mean age of 66 years old, with a diagnosis of symptomatic peripheral arterial disease (PAD). The control group was treated with open revascularization using ePTFE graft, whereas the study group was treated with the DES Zilver PTX. Both groups were evaluated immediately, at 3 and 6 months. Results: In comparison, both methods were effective to treat PAD. DES Zilver PTX reduced hospitalization length and had a higher tendency to increase functional class. Primary patency was $87 \%$ vs $93 \%$ and secondary patency was $93 \%$ vesus $100 \%$ for open revascularization

Correspondencia:

${ }^{*}$ César Nuño Escobar

E-mail: medicovascular@yahoo.com
Disponible en internet: 20-09-2019 Rev Mex Angiol. 2019;47(3):6-10 www.RMAngiologia.com

0377-4740/@ 2019 Sociedad Mexicana de Angiología y Cirugía Vascular y Endovascular, A.C. Published by Permanyer México. This is an open access article under the CC BY-NC-ND license (http://creativecommons.org/licenses/by-nc-nd/4.0/). 
and DES respectively. Conclusions: Both methods are effective to treat $P A D$ in the infrainguinal segment. Our results tend to favor DES over open revascularization with synthetic graft.

Key words: Drug eluting stent. Zilver PTX. Synthetic graft. Synthetic revascularization.

\section{Introducción}

La enfermedad arterial periférica (EAP) es el resultado de un proceso degenerativo crónico, que se caracteriza por el acúmulo de placas de ateroma de la íntima en los vasos $^{1,2}$. La prevalencia mundial es desconocida, sin embargo, se estiman de 8 a 12 millones de pacientes afectados en los EE.UU. ${ }^{3,4}$. Cada año, más de 100,000 pacientes con diagnóstico de enfermedad arterial periférica serán sometidos a algún procedimiento de revascularización ${ }^{5}$. La derivación abierta continúa siendo una técnica efectiva, ya que la utilización de vena autóloga tiene una permeabilidad de hasta el $70 \%$ a 4 años ${ }^{6,7}$, mientras que con el uso de stent medicado Zilver PTX se reporta de un $89.9 \%$ a 1 año, $0^{7,8}$. La derivación abierta se asocia a mayor morbimortalidad ${ }^{9,10}$, incremento en días de estancia intrahospitalaria, enfermedades nosocomiales y costo total en comparación a la vía endovenosa $^{11}$. El stent Zilver PTX es un stent autoexpandible liberador de medicamento, cuenta con $3 \mathrm{mg} / \mathrm{mm}^{3}$ de paclitaxel libre de polímero recubierto en su superficie, y disminuye el desarrollo de hiperplasia intimal para lograr menores índices de reestenosis en comparación a la angioplastia con balón o a los stents desnudos ${ }^{12-14}$.

El objetivo de este estudio es analizar la permeabilidad y presencia de complicaciones del stent medicado Zilver PTX en comparación a la derivación femoropoplítea abierta con injerto sintético de Politetrafluoroetileno (PTFe).

\section{Métodos}

Se trata de un estudio retrospectivo analítico, realizado de julio de 2014 a enero de 2015, que comprendió 30 pacientes con enfermedad femoropoplítea sintomática candidatos a cirugía de revascularización. Fue aprobado por el Comité de Ética e Investigación de la Unidad Médica de Alta Especialidad (UMAE) del Centro Médico Nacional de Occidente (CMNO).

Mediante aleatorización se establecieron dos grupos: un grupo control para 15 pacientes tratados mediante derivación abierta con injerto de PTFe y un grupo de estudio para 15 pacientes manejados vía endovascular utilizando el stent medicado Zilver PTX. Se realizaron tres mediciones en todos los pacientes (prequirúrgicas, posquirúrgicas y a los 6 meses), y valoraron la edad, sexo, estadificación de Rutherford, índice tobillo brazo
Tabla 1. Reporte de edad y sexo para ambos grupos

\begin{tabular}{|l|c|}
\hline Variable & $\begin{array}{c}\text { Valor } \\
(\mathbf{n}=30)\end{array}$ \\
\hline Edad, años & $66 \pm 7$ \\
\hline $\begin{array}{c}\text { Sexo, } n(\%) \\
\text { Masculino } \\
\text { Femenino }\end{array}$ & $20(67 \%)$ \\
\hline
\end{tabular}

Tabla 2. Comparativo de mediciones basales de variables a comparar entre ambos grupos

\begin{tabular}{|l|c|c|c|}
\hline Variable & $\begin{array}{c}\text { Derivación } \\
\text { abierta }\end{array}$ & $\begin{array}{c}\text { Colocación } \\
\text { del stent }\end{array}$ & Valor p \\
\hline $\begin{array}{l}\text { Índice } \\
\text { tobillo-brazo basal }\end{array}$ & $0.50(0.40-0.57)$ & $0.88(0.39-2)$ & 0.46 \\
\hline $\begin{array}{l}\text { TASC } \\
\text { preoperatorio }\end{array}$ & & & \\
(estadio) & $6(40)$ & $8(53)$ & 0.14 \\
B & $2(13)$ & $5(34)$ & \\
C & $7(47)$ & $2(13)$ & \\
D & & & \\
\hline Estadificación & & & \\
Rutherford basal & & & \\
3 & $6(40)$ & $8(53)$ & 0.32 \\
4 & $4(27)$ & $1(7)$ & \\
5 & $4(27)$ & $6(40)$ & \\
6 & $1(7)$ & 0 & \\
\hline
\end{tabular}

(ITB), TASC II (Consenso Multidisciplinario para el Manejo de la Enfermedad Arterial Periférica), presencia de comórbidos e índice tabáquico. Dichos datos fueron captados mediante un cuestionario en consulta externa del Instituto Mexicano del Seguro Social, UMAE, CMNO. Se realizó ultrasonograma Doppler a los 6 meses para valorar permeabilidad.

\section{Resultados}

Se estudiaron 30 pacientes, 15 para cada grupo, y ambos grupos fueron similares. La edad promedio fue de $66 \pm 7$ años. El $67 \%$ de los pacientes eran del sexo masculino. Las características de la muestra en general se presentan en la tabla 1.

Las principales comorbilidades de la muestra se presentan en la figura 1, donde 27 pacientes (90\%) tienen diagnóstico de Hipertensión Arterial Sistémica (HAS), 


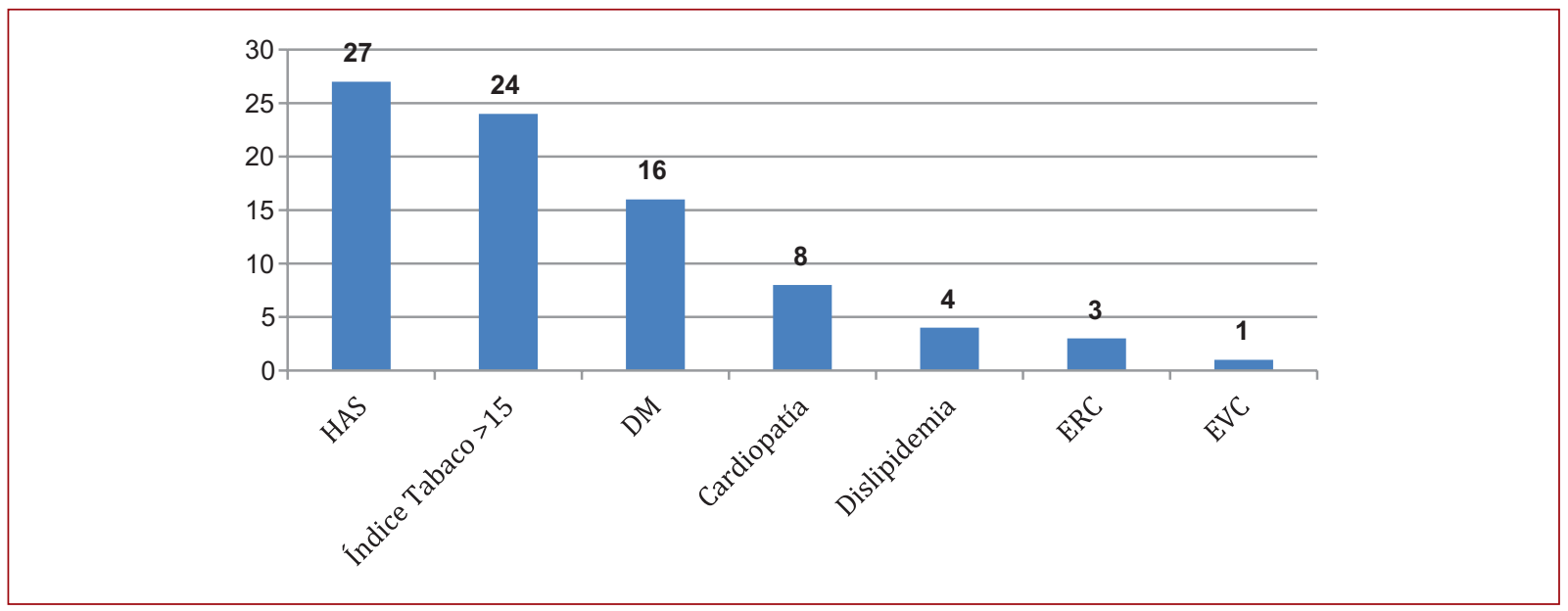

Figura 1. Presencia de comórbidos en el total de pacientes.

DM: diabetes mellitus; ERC: enfermedad renal crónica; EVC: evento vascular cerebral; HAS: Hipertensión Arterial Sistémica.

Tabla 3. Comparativa de variables a 6 meses

\begin{tabular}{|l|c|c|c|}
\hline Variable & $\begin{array}{c}\text { Derivación } \\
\text { abierta }\end{array}$ & $\begin{array}{c}\text { Colocación del } \\
\text { stent }\end{array}$ & Valor p \\
\hline $\begin{array}{l}\text { Índice } \\
\text { tobillo-brazo a } \\
\text { los 6 meses }\end{array}$ & $0.76(0.60-0.92)$ & $0.80(0.70-2.00)$ & 0.42 \\
\hline $\begin{array}{l}\text { Permeabilidad } \\
\text { primaria }\end{array}$ & $13(87 \%)$ & $14(93 \%)$ & 0.50 \\
\hline $\begin{array}{l}\text { Permeabilidad } \\
\text { secundaria }\end{array}$ & $14(93 \%)$ & $15(100 \%)$ & 0.30 \\
\hline $\begin{array}{l}\text { Estadificación } \\
\text { Rutherford 6 }\end{array}$ & & & \\
meses & & & \\
0 & $4(27)$ & $3(30)$ & 0.63 \\
1 & $4(27)$ & $3(30)$ & \\
2 & $3(20)$ & $5(33)$ & \\
3 & $2(12)$ & $4(27)$ & \\
\hline 5 & $1(7)$ & 0 & \\
6 & $1(7)$ & 0 & \\
\hline
\end{tabular}

16 pacientes (53\%), diabetes mellitus, y 8 pacientes (26.6\%) tienen alguna cardiopatía. Del total de pacientes, 24 (80\%) presentaron un índice de tabaco mayor a 15 paquetes por año, con una media de 35.5 paquetes anuales. Solo 5 pacientes no contaban con antecedente de tabaquismo, sin embargo, todos los pacientes sin antecedente de tabaquismo, tenían diagnóstico de diabetes mellitus (Fig. 1).

En la tabla 2 se muestra que ni el ITB, ni la estadificación Rutherford, ni tampoco la estadificación de TASC tenían diferencias estadísticamente significativas al inicio del estudio (Tabla 2). Al final del estudio
Tabla 4. Comparativa de resultados a 6 meses para ambos grupos

\begin{tabular}{|l|c|c|c|}
\hline Variable & $\begin{array}{c}\text { Derivación } \\
\text { abierta }\end{array}$ & $\begin{array}{c}\text { Colocación } \\
\text { del stent }\end{array}$ & Valor p \\
\hline Edad, años & $66 \pm 7$ & $66 \pm 7$ & 0.99 \\
\hline Días de hospitalización & $3.6(2-7)$ & $2.2(2-4)$ & 0.003 \\
\hline $\begin{array}{l}\text { Salvamento, n (\%) } \\
\text { Sin amputación }\end{array}$ & $\begin{array}{c}13(88) \\
1(6)\end{array}$ & $\begin{array}{c}13(88) \\
0\end{array}$ & 0.51 \\
$\begin{array}{l}\text { Amputación mayor } \\
\text { Amputación menor }\end{array}$ & $\begin{array}{c}1(6) \\
2(12)\end{array}$ & \\
\hline $\begin{array}{l}\text { Efectos adversos, n (\%) } \\
\text { Sí } \\
\text { No }\end{array}$ & $\begin{array}{c}4(27) \\
11(73)\end{array}$ & $\begin{array}{c}2(13) \\
13(87)\end{array}$ & 0.36 \\
\hline
\end{tabular}

tuvieron una mejoría mayor en el grupo endovascular que en el grupo de derivación abierta, sin logar una diferencia estadística ( $p=0.42 ; p=63$ ) (Tabla 3).

Los días de hospitalización son menores para el grupo endovascular (2.26) en comparación a la derivación abierta (3.6), con diferencia significativa ( $p=0.003$ ).

Como complicaciones, para el grupo de derivación abierta se presentaron dos dehiscencias de herida quirúrgica. Para la terapia endovascular se presentó una angina estable transoperatoria que se resolvió con la administración de analgesia y una presencia de hematoma femoral no expansivo que se resolvió a la compresión mecánica (Tabla 4). Un paciente requirió amputación mayor en la derivación abierta, mientras que en la colocación del stent ninguno $(p=0.51)$. La permeabilidad es similar para ambas técnicas, sin encontrar diferencia 
Tabla 5. Comparativo basal y a 6 meses de las variables de interés para ambos grupos

\begin{tabular}{|c|c|c|c|c|c|}
\hline \multirow[t]{2}{*}{ Variable } & \multicolumn{2}{|c|}{ Derivación abierta } & \multicolumn{2}{|c|}{ Colocación del stent } & \multirow[t]{2}{*}{ Valor p } \\
\hline & Inicial & 6 meses & Inicial & 6 meses & \\
\hline Índice tobillo-brazo & $\begin{array}{c}0.50 \\
(0.40-0.57)\end{array}$ & $\begin{array}{c}0.76 \\
(0.60-0.92)\end{array}$ & $\begin{array}{c}0.88 \\
(0.39-2)\end{array}$ & $\begin{array}{c}0.80 \\
(0.70-2.00)\end{array}$ & 0.0001 \\
\hline $\begin{array}{l}\text { Estadio Rutherford n (\%) } \\
0 \\
1 \\
2 \\
3 \\
4 \\
5 \\
6\end{array}$ & $\begin{array}{c}0 \\
0 \\
0 \\
6(40) \\
4(27) \\
4(27) \\
1(7)\end{array}$ & $\begin{array}{l}4(27) \\
4(27) \\
3(20) \\
2(12) \\
0 \\
1(7) \\
1(7)\end{array}$ & $\begin{array}{c}0 \\
0 \\
0 \\
8(53) \\
1(7) \\
6(40) \\
0\end{array}$ & $\begin{array}{c}3(30) \\
3(30) \\
5(33) \\
4(27) \\
0 \\
0 \\
0\end{array}$ & 0.001 \\
\hline $\begin{array}{l}\text { Permeabilidad } \\
\text { Primaria/secundaria } \mathrm{n}(\%)\end{array}$ & $13(87)$ & $14(93)$ & 14 (93) & $15(100)$ & 0.15 \\
\hline
\end{tabular}

estadística $(p=0.15)$ (Tabla 5). Para el grupo tratado mediante derivación abierta, se presentó oclusión de injerto en 2 pacientes con enfermedad Rutherford 6, y fueron sometidos ambos a trombectomía. Un paciente con colocación de stent tuvo un episodio de estenosis, manejado con angioplastia intra-stent, y recobró permeabilidad. Cabe resaltar que los 3 pacientes reinervenidos refirieron no haber suspendido el hábito tabáquico.

\section{Discusión}

Nuestra primera limitante fue el tiempo de vigilancia de los pacientes, por lo que aclaramos que se trató de un estudio de resultados inmediatos, especificando la necesidad de realizar un estudio de vigilancia a largo plazo en ambos grupos para determinar los resultados a largo plazo. Los factores predisponentes, como el sexo masculino, edad avanzada y tabaquismo, se presentaron en este estudio como los principales para el desarrollo de EAP en el sector femoropoplíteo, coincidiendo con lo reportado por Biancari ${ }^{13}$. El $90 \%$ de los pacientes de nuestro estudio tenían diagnóstico de hipertensión arterial sistémica, y se definió como el principal factor de riesgo asociado a EAP en el sector femoropoplíteo. El índice tabáquico se presentó como el segundo factor de riesgo más importante: el $80 \%$ de los pacientes estudiados consumían más de 15 paquetes anuales. El 53.3\% contaban con diagnóstico de diabetes mellitus, que estaba presente en todos los pacientes con ausencia de tabaquismo. Dichas cifras proyectan un panorama epidemiológico en nuestra población, con el cual podremos reforzar la importancia de una medicina preventiva eficaz. Ambos grupos presentaron una mejoría de ITB y de clase funcional (evaluada por escala de Rutherford) con resultados estadísticamente significativos ( $p=0.0001$ ), lo que nos muestra que ambas técnicas son efectivas para el manejo de la EAP del sector femoropoplíteo. Comparando ambas técnicas, la única diferencia significativa $(p=0.003)$ se presentó en la reducción en días de estancia intrahospitalaria para el grupo endovascular. Esto se traduce en reducción de costos hospitalarios, disminución en riesgo de infecciones nosocomiales y una reincorporación más temprana a la vida laboral. Nuestras cifras se asemejan a las reflejadas en los estudios publicados por Kudo, et al. ${ }^{15} \mathrm{y}$ Goshima, et al. ${ }^{16}$. Todas las derivaciones abiertas se realizaron con injerto de PTFe, para el cual, Eickhoff, et al. ${ }^{17}$ reportaron cifras de permeabilidad del $53 \%$ a 3 meses. Green ${ }^{18}$ hizo un seguimiento a 4 años, con permeabilidad del $60 \%$. Nuestros resultados superaron estas cifras, con permeabilidad primaria del $87 \%$ y secundaria del $94 \%$. El grupo tratado con Zilver PTX logró el $94 \%$ y el $100 \%$ de permeabilidad primaria y secundaria, respectivamente. Dake, et al. ${ }^{2}$ reportan una permeabilidad del $83 \%$ a los 2 años, cifra similar a la obtenida en nuestro centro. Si bien no se presentan diferencias estadísticamente significativas, el resultado es de valor, ya que no existe superioridad con conducto sintético. La realización del ITB se presenta como una prueba útil para valorar de manera indirecta el resultado y permeabilidad de los procedimientos de revascularización. Una limitante encontrada durante la realización de nuestro estudio fue la presencia de esclerosis de Monckeberg durante la prueba en pacientes diabéticos, otorgando resultados en cifras tan altas como 2. De acuerdo al consenso TASC II $^{19}$, las lesiones del sector femoropoplíteo $C$ y $D$ pueden tratarse por vía endovascular según el criterio del cirujano vascular ${ }^{20}$. Okhi, et al. manejan 
este tipo de lesiones utilizando stent medicado Zilver PTX, con adecuada permeabilidad a los 2 años ${ }^{21}$. En nuestro estudio se trata a 7 de 15 pacientes con stent medicado que presentan lesiones TASC $C$ y $D$, con adecuados resultados a corto plazo. Para valorar la clase funcional utilizamos la clasificación de Rutherford, y valoramos la distancia de la marcha y la pérdida de tejidos. Ambos grupos mostraron mejoría de clase funcional, sin encontrar una diferencia estadísticamente significativa. Existe una clara tendencia a mayor beneficio de clase funcional con el uso de stent medicado que con derivación con conducto sintético. La vigilancia a largo plazo y aumento de tamaño de la muestra son necesarias para determinar diferencia entre ambos grupos.

\section{Conclusiones}

La terapia endovascular logra reducir de manera significativa los días de estancia intrahospitalaria, lo que reduce los costos hospitalarios y consigue la reincorporación más temprana a la vida laboral. Nuestra población presenta similitud en los factores de riesgo asociados al desarrollo de EAP femoropoplítea a lo reportado en la literatura mundial, a pesar de la alta prevalencia de diabetes mellitus en nuestro país. Ambas técnicas son efectivas para el salvamento de extremidades, por lo que las dos son recomendables para el manejo de la EAP en el sector femoropoplíteo. En la clasificación TASC no existió diferencia entre los grupos. Se lograron tratar lesiones $C$ y $\mathrm{D}$ con el stent medicado Zilver PTX con adecuada permeabilidad a los 6 meses. No se encontraron evidencias estadísticas de que una técnica fuera mejor que otra, sin embargo, en la colocación de stent se puede observar una mayor mediana y cuartiles del ITB. La realización de estudios comparativos entre ambas técnicas es esencial para determinar el estándar de oro en el manejo de la EAP en el sector femoropoplíteo.

\section{Conflicto de intereses}

Los autores declaran que no existe conflicto de intereses. La totalidad del estudio fue realizada con material de la UMAE del CMNO.

\section{Responsabilidades éticas}

Protección de personas y animales. Los autores declaran que para esta investigación no se han realizado experimentos en seres humanos ni en animales.
Confidencialidad de los datos. Los autores declaran que han seguido los protocolos de su centro de trabajo sobre la publicación de datos de pacientes.

Derecho a la privacidad y consentimiento informado. Los autores declaran que en este artículo no aparecen datos de pacientes.

\section{Bibliografía}

1. Cronenwett J, Johnston W. Rutherford's Vascular Surgery. 7. a ed. Filadelfia: Editorial Saunders; 2010.

2. Varu VN, Hogg M, Kibbe M. Review articles: Critical limb ischemia. J Vasc Surg. 2012;51(1):230-41.

3. Criqui MH, Fronek A, Barrett-Connor E, Klauber MR, Gabriel S, Goodman D. The prevalence of peripheral arterial disease in a defined population. Circulation. 1985;71:510-15.

4. Hirsch A, Criqui MH, Treat-Jacobson D, Regensteiner JG, Creager MA, Olin JW, et al. Peripheral arterial disease detection, awareness and treatment in primary care. JAMA. 2001;286:1317-24. Hirsch AT, Hartman L, Town RJ, Virnig BA. National health care costs of peripheral arterial disease in the Medicare population. Vascular Medicine. 2008;13:209-15.

5. Cronenwett J, Johnston W. Rutherford's Vascular Surgery. 8. ${ }^{a}$ ed. Filadelfia: Editorial Saunders, 2014.

6. Heyligers JM, Verhagen HJ, Rotmans JI, Weeterings C, de Groot PG, Moll FL, et al. Heparin immobilization reduces thrombogenicity of small-caliber expanded polytetrafluoroethylene grafts. J Vasc Surg. 2006;43:587-91.

7. Dake MD, Ansel GM, Jaff MR, Ohki T, Saxon RR, Smouse HB, et al. Paclitaxel-eluting stents show superiority to balloon angioplasty and bare metal stents in femoropopliteal disease: twelve- month Zilver PTX randomized study results. Circ Cardiovasc Interv. 2011;4:495-504.

8. Laird JR, Katzen BT, Scheinert D, Lammer J, Carpenter J, Buchbinder M, et al. Nitinol stent implantation versus balloon angioplasty for lesions in the superficial femoral artery and proximal popliteal artery: twelve-month results from the RESILIENT randomized trial. Circ Cardiovasc Interv. 2010;3:267-76.

9. Beard JD. Wich is the best revascularization for critical limb ischemia: Endovascular or open surgery? J Vasc Surgery. 2008;21(4):210-6.

10. Marek JM, Mills JL. Risk factor assessment and indications for reconstruction. In Mills JL, editor: Management of chronic lower limb ischemia, Arnold, pp 30-44, London, 2000.

11. Adam DJ. Bypass versus angioplasty in severe ischaemia of the leg (BASIL): multicentre, randomised controlled trial. Lancet. 2005;366(9501):1925-34.

12. Bradbury AW, Adam DJ, Bell J, Forbes JF, Fowkes FG, Gillespie I, et al.: Bypass versus Angioplasty in Severe Ischaemia of the Leg (BASIL) trial: An intention-to-treat analysis of amputation-free and overall survival in patients randomized to a bypass surgery-first or a balloon angioplasty-first revascularization strategy. J Vasc Surg. 2010 Dec;52(6):1751.

13. Biancari $F$. Meta-analysis of the prevalence, incidence and natural history of critical limb ischemia. J Cardiovasc Surg. 2013;54(6):663-9.

14. Krankenberg $H$, Sclüter $M$, Steinkamp HJ, Bürgelin $K$, Scheinert $D$, Schulte $\mathrm{KL}$, et al. Nitinol stent implantation versus percutaneous transluminal angioplasty in superficial femoral artery lesions up to $10 \mathrm{~cm}$ in length: the femoral artery stenting trial (FAST). Circulation. 2007;116:285-92.

15. Kudo T, Chandra FA, Kwun WH, Haas BT, Ahn SS. Changing pattern of surgical revascularization for critical limb ischemia over 12 years: endovascular vs. open bypass surgery. J Vasc Surg. 2006;44:304-13.

16. Goshima KR, Mills JL Sr, Hughes JD. A new look at outcomes after infrainguinal bypass surgery: traditional reporting standards systematically underestimate the expenditure of effort required to attain limb salvage. $J$ Vasc Surg. 2004;39:330-5.

17. Eickhoff $\mathrm{JH}$, Broomé A, Ericsson B, Hansen HJB, Kordt KF, Mouritzen $\mathrm{C}$ et al.: Four years' results of a prospective, randomized clinical trial comparing polytetrafluoroethylene and modified human umbilical vein for below-knee femoropopliteal bypass. J Vasc Surg. 1987;6:506-11.

18. Green RM, Abbott W, Matsumoto T, Wheeler JR, Miller N, Veith FR, et al.: Prosthetic above-knee femoropopliteal bypass grafting: five-year results of a randomized trial. J Vasc Surg. 2000;31:417-25.

19. Jaff MR, White CJ, Hiatt WR, Fowkes GR, Dormandy J, Razavi M, et al. An Update on Methods for Revascularization and Expansion of the TASC Lesion Classification to Include Below-the-Knee Arteries: A Supplement to the Inter-Society Consensus for the Management of Peripheral Arterial Disease (TASC II). Vascular Medicine. 2015;22:657-71.

20. Leopardi M, Houballah R, Becquemin JP. Effectiveness of Zilver PTX eluting stent in TASC C/D lesions and restenosis. J Cardiovasc Surg (Torino). 2014 April;55(2):229-34.

21. Okhi T, Yokoi H, Kichikawa T, snyder SA, Ragheb AO, O'Leary EE, Jaff MR, Ansel GM, Dake MD. Two-year analysis of the Japanese cohort from the Zilver PTX randomized controlled trial supports the validity of multinational clinical trials. J Endovasc Ther. 2014 Oct;21(5):644-53. 